\title{
Ultrasensitive PbS quantum-dot-sensitized InGaZnO hybrid photoinverter for near-infrared detection and imaging with high photogain
}

\author{
Do Kyung Hwang ${ }^{1,2,8}$, Young Tack Lee ${ }^{1,8}$, Hee Sung Lee ${ }^{3}$, Yun Jae Lee ${ }^{1,4}$, Seyed Hossein Shokouh ${ }^{3}$, \\ Ji-hoon Kyhm ${ }^{1}$, Junyeong Lee ${ }^{3}$, Hong Hee Kim ${ }^{1}$, Tae-Hee Yoo ${ }^{1}$, Seung Hee Nam ${ }^{3}$, Dong Ick Son ${ }^{5}$, \\ Byeong-Kwon Ju${ }^{4}$, Min-Chul Park ${ }^{6}$, Jin Dong Song ${ }^{1}$, Won Kook Choi ${ }^{2,7}$ and Seongil $\mathrm{Im}^{3}$
}

Lead sulfide ( $\mathrm{PbS}$ ) quantum dots (QDs) have great potential in optoelectronic applications because of their desirable characteristics as a light absorber for near-infrared (NIR) photodetection. However, most PbS-based NIR photodetectors are two-terminal devices, which require an integrated pixel circuit to be practical photosensors. Here we report on PbS QD/indium gallium zinc oxide (InGaZnO, IGZO) metal oxide semiconductor hybrid phototransistors with a photodetection capability between 700 and $1400 \mathrm{~nm}$, a range that neither conventional Si nor InGaAs photodetectors can cover. The new hybrid phototransistor exhibits excellent photoresponsivity of over $10^{6} \mathrm{~A} \mathrm{~W}^{-1}$ and a specific detectivity in the order of $10^{13}$ Jones for NIR (1000 nm) light. Furthermore, we demonstrate an NIR $(1300 \mathrm{~nm})$ imager using photogating inverter pixels based on PbS/IGZO phototransistors at an imaging frequency of $1 \mathrm{~Hz}$ with a high output voltage photogain of $\sim 4.9 \mathrm{~V}$ ( $99 \%)$. To the best of our knowledge, this report demonstrates the first QD/metal oxide hybrid phototransistor-based flat panel NIR imager. Our hybrid approach using QD/metal oxide paves the way for the development of gate-tunable and highly sensitive flat panel NIR sensors/ imagers that can be easily integrated.

NPG Asia Materials (2016) 8, e233; doi:10.1038/am.2015.137; published online 8 January 2016

\section{INTRODUCTION}

Over the past several years, various chalcogenide or metal oxide colloidal quantum dots (QDs) have been synthesized and developed for optoelectronic device applications such as light emitting diodes, ${ }^{1-4}$ photodetectors $^{5-12}$ and photovoltaic cells. ${ }^{13-18}$ Among the various types of QDs, lead sulfide (PbS) QD has been extensively studied because it is a promising material for collecting sunlight energy ${ }^{14,15}$ or for near-infrared (NIR) photodetection. ${ }^{8,19}$ Recently, the commercial interest in NIR detection has been growing due to the potential for various imaging applications including optical tomography for biological investigations, process monitoring and night vision. ${ }^{19}$ Sensitivity to the spectral region between 1000 and $1800 \mathrm{~nm}$ without significant water absorption loss is of particular importance. ${ }^{19}$ Conventional and cost-effective Si-based detectors cannot be considered for use in this wavelength region; InGaAs-based detectors are widely used instead but at a higher cost. The energy bandgap of
$\mathrm{PbS}$ QD can be easily tuned to fit in this wavelength range, and PbS QD-based photoconductors ${ }^{6,7}$ or PbS QD/organic semiconductor hybrid photodiodes ${ }^{5,19}$ have been reported. However, these two-terminal devices need to be integrated onto an active matrix transistor backplane as an integrated pixel circuit for more practical photosensing applications. ${ }^{19,20}$

In addition, amorphous metal oxide semiconductor-based thin-film transistors (TFTs) have attracted considerable attention as an emerging technology to replace amorphous and polycrystalline $\mathrm{Si}$ TFTs because of their high optical transparency, amorphous microstructure, high electron mobility, low off-current level and low processing temperatures; these advantages may make large-area and low-cost microelectronic circuit systems possible. ${ }^{21-27}$ Considerable effort has been devoted to optimize the fabrication process and to improve the device performance and reliability. ${ }^{27}$ As a consequence, amorphous metal oxide semiconductor indium gallium zinc oxide

${ }^{1}$ Center for Opto-Electronic Materials and Devices, Post-Silicon Semiconductor Institute, Korea Institute of Science and Technology (KIST), Seoul, South Korea; ${ }^{2}$ Department of Nanomaterials and Nano Science, Korea University of Science and Technology (KUST), Daejun, South Korea; ${ }^{3}$ Institute of Physics and Applied Physics, Yonsei University, Seoul, South Korea; ${ }^{4}$ Display and Nanosystem Laboratory, School of Electrical Engineering, Korea University, Seoul, South Korea; ${ }^{5}$ Soft Innovative Materials Research Center, Korea Institute of Science and Technology (KIST), Jeonbuk, South Korea; ${ }^{6}$ Sensor System Research Center, Korea Institute of Science and Technology (KIST), Seoul, South Korea and ${ }^{7}$ Materials and Life Science Research Division, Korea Institute of Science and Technology (KIST), Seoul, South Korea

8These authors contributed equally to this work.

Correspondence: Dr DK Hwang, Center for Opto-Electronic Materials and Devices, Korea Institue of Science and Technology, Hwarangno 14-gil 5, Seongbuk-gu, Seoul 02792, South Korea.

E-mail: dkhwang@kist.re.kr

or Dr WK Choi, Materials and Life Science Research Division, Korea Institute of Science and Technology (KIST), Seoul 02792, South Korea.

E-mail: wkchoi@kist.re.kr

or Professor S Im, Institute of Physics and Applied Physics, Yonsei University, Seoul 03722, South Korea.

Email: semicon@yonsei.ac.kr

Received 29 July 2015; revised 22 October 2015; accepted 25 October 2015 
(InGaZnO, IGZO) TFTs are very close to being commercialized in active matrix backplanes as a pixel driver for high-resolution liquid crystal displays and organic light-emitting diodes display. In addition to flat panel displays, IGZO TFT-based active matrix backplane can be extended to other integrated electronic and optoelectronic systems. ${ }^{20}$ Recently, IGZO-based phototransistor and integrated photosensor array have been reported. ${ }^{20}$ Nevertheless, the performance of metal oxide semiconductors as photoactive layers has been limited in the ultraviolet wave band due to their wide energy bandgap (larger than $3.0 \mathrm{eV})$.

A hybrid approach that combines the above two classes of PbS QDs as the NIR light absorber and IGZO as the photogenerated charge acceptor/transport semiconductor can provide a means to circumvent the aforementioned issues. The PbS QD can be functionalized directly onto the surface of the IGZO TFT to create a new bi-functional optoelectronic device: a gate tunable, highly sensitive and easily integrated NIR-sensing three-terminal phototransistor. The device concept and the material have been devised, and the corresponding preliminary results have been reported in our previous work. ${ }^{28}$ Recently, the PbS QD has been combined with two-dimensional graphene nanosheets prepared by mechanical exfoliation or chemical vapor deposition to fabricate phototransistors for the visible or NIR region. ${ }^{29-31}$ However, graphene transistors show very poor on/off current ratio, which is a critical problem because switching and modulation are basic functions of a transistor. Other than graphene, a $\mathrm{PbS} \mathrm{QD} / \mathrm{MoS}_{2}$ hybrid phototransistor has been reported, which shows good phototransistor performance with an acceptable on/off current ratio. ${ }^{32}$ Despite the recent progress, integration of such transistors onto the active matrix backplane on large-area glass or plastic substrates is still challenging.

In this work, PbS QD sensitized IGZO hybrid phototransistor was fabricated through a facile spin-coating process, in which colloidal $\mathrm{PbS}$ QDs were formed on the top of prefabricated IGZO TFT arrays on glass substrates. This hybrid phototransistor responded to NIR light up to $1400 \mathrm{~nm}$. The photogenerated electrons from the $\mathrm{PbS}$ sensitized layer could be easily transferred to the conduction band of the IGZO layer, leading to significant negative shifts of the threshold voltage $\left(V_{\text {th }}\right)$ in the IGZO TFT. As a result, the hybrid phototransistor exhibited excellent photoresponsivity of over $10^{6} \mathrm{~A} \mathrm{~W}^{-1}$ and a specific detectivity on the order of $10^{13}$ Jones for NIR $(1000 \mathrm{~nm})$ light. A photogating resistive load inverter was implanted by connecting a unit phototransistor to an external load resistor that showed pronounced output voltage signals with a high output voltage photogain of $\sim 4.9 \mathrm{~V}$ at close to $99 \%$. Using this photogating inverter as the basic unit, a prototype NIR $(1300 \mathrm{~nm})$ imager was demonstrated.

\section{EXPERIMENTAL PROCEDURES}

\section{Device fabrication}

The IGZO TFT array with a bottom gate and top contact structure was fabricated on glass substrate. A Mo gate electrode was deposited by dc sputtering. An $\mathrm{SiO}_{2}(350 \mathrm{~nm})$ gate dielectric layer was then deposited by plasma-enhanced chemical vapor deposition, and the capacitance density was measured to be $8.6 \mathrm{nF} \mathrm{cm}^{-2}$. Amorphous IGZO $\left(\mathrm{Ga}_{2} \mathrm{O}_{3}: \mathrm{In}_{2} \mathrm{O}_{3}\right.$ : $\mathrm{ZnO}=1: 1: 1 \mathrm{~mol} \%)$ active layers $(30 \mathrm{~nm})$ were deposited by a radio frequency sputtering system using a power of $80 \mathrm{~W}$ at a working pressure of $2 \mathrm{~m}$ Torr and a mixture of $\mathrm{Ar} / \mathrm{O}_{2}$ (80/20 s.c.c.m). Then, an IGZO thin film was patterned by wet-etching to define the channel and was subsequently annealed at $200{ }^{\circ} \mathrm{C}$ for $2 \mathrm{~h}$ under ambient conditions. The Au/Ti source/drain electrodes were then prepared by dc sputtering and patterned by a conventional lift-off process. Colloidal PbS QDs (Nanodot-PbS 1300) with the first excitation peak at $\sim 1320 \mathrm{~nm}$ was purchased from (QD Solution Co., Ltd, Daejun, Korea), and the QDs were capped with oleic acid (OA) ligand and dispersed in toluene with a concentration of $100 \mathrm{mg} \mathrm{ml}^{-1}$. The original solution was further diluted with toluene to a concentration of $5 \mathrm{mg} \mathrm{ml}^{-1}$. The $\mathrm{PbS}$ QDs layers were deposited by spin coating at 500 r.p.m. for $3 \mathrm{~s}$ followed by 2000 r.p.m. for $40 \mathrm{~s}$ in a nitrogen-filled glove box; they were then annealed at $110^{\circ} \mathrm{C}$ for $30 \mathrm{~min}$ to remove any residual solvent. The longer OA ligands were replaced with the shorter ethanedithiol (EDT) ligands by the ligand-exchange process as follows: the EDT solutions were dissolved in methanol or acetonitrile with a concentration of $0.1 \mathrm{wt} \%$. As-prepared $\mathrm{PbS}$ films were fully covered with drops of EDT solutions, stayed for $1 \mathrm{~min}$, dried by spin coating at 2000 r.p.m. for $60 \mathrm{~s}$, rinsed with pure methanol or acetonitrile and finally annealed at $110^{\circ}$ $\mathrm{C}$ for $30 \mathrm{~min}$ in a vacuum chamber. An amorphous fluoropolymer CYTOP (Asahi Glass, Tokyo, Japan, $100 \mathrm{~nm}$ ) film was deposited on the top of the devices by spin coating at 2000 r.p.m. for $60 \mathrm{~s}$ and annealed at $100{ }^{\circ} \mathrm{C}$ for $20 \mathrm{~min}$ in a nitrogen-filled glove box. This CYTOP film was used as a passivation layer because the PbS QD capped with the EDT ligand is vulnerable to degradation in ambient air. A CYTOP film is better than conventional polymer films in terms of water and oxygen permeability. In addition, CYTOP is highly transparent in the NIR spectrum region (over 90\%), which is an appropriate window for an NIR detector.

\section{Material, electrical and photoelectric characterization}

High-resolution transmission electron microscopy (JEOL, Tokyo, Japan, JEM $2100 \mathrm{~F}$ ) was used to analyze the microstructure and uniformity in the PbS QD films. The Fourier transform infrared (Thermo Nicolet iS10, Waltham, MA, USA) and absorption (JASCO V-670 spectrophotometer, JASCO, Easton, MD, USA) measurements were carried out to evaluate the degree of ligand exchange process in the PbS QD films. All current-voltage $(I-V)$ and NIR photodetecting properties of the hybrid phototransistors were measured with a photoexcited charge collection spectroscopy system that consisted of a semiconductor parameter analyzer (HP 4155C, Agilent Technologies, Santa Clara, CA, USA), a light source of $500 \mathrm{~W} \mathrm{Hg}(\mathrm{Xe})$ arc lamp, a gratings monochromator covering the spectral range of $1500-700 \mathrm{~nm}$, and an optical fiber (core diameter of $200 \mu \mathrm{m}$ ), enabling the system to deliver light at specific wavelength ranges. In order to calculate the noise equivalent power (NEP) and the specific detectivity $\left(D^{*}\right)$, the noise power spectral densities were measured with an SR 570 lownoise-current preamplifier (Stanford Research System (SRS), Sunnyvale, CA, USA) and an Advantest R9211B digital spectrum analyzer (Advantest Corporation, Tokyo, Japan).

\section{RESULTS AND DISCUSSION}

\section{Device schematic and the ligand-exchange process}

Figures $1 \mathrm{a}$ and $\mathrm{b}$ show the three-dimensional (3D) scheme of the $\mathrm{PbS}$ QD/IGZO hybrid phototransistor and the corresponding cross-sectional high-resolution transmission electron microscopy images, respectively. The Mo-gate electrode, the $\mathrm{SiO}_{2}$ dielectric layer, the IGZO channel and the $\mathrm{PbS} \mathrm{QD}$ layers comprising the phototransistor were clearly observed. As can be seen in the magnified transmission electron microscopy images with the red and yellow dotted line squares, the $\mathrm{PbS}$ film consisted of numerous $\mathrm{PbS}$ QDs with an average diameter of $4-5 \mathrm{~nm}$, which were uniformly distributed on the IGZO layer, with the PbS QD layer thickness estimated to be $\sim 6.5 \mathrm{~nm}$ from transmission electron microscopy results.

The PbS QDs were generally capped with the OA ligand with long alkyl chains during their synthesis process to enhance the solution processability and stability. However, the insulating nature of the long alkyl chains is likely to hinder the charge carrier transport within the $\mathrm{PbS}$ QD layer ${ }^{17,33}$ or block the charge transfer between PbS QD and the active channel. ${ }^{30,31}$ Several studies on ligand exchanges have been reported. ${ }^{14,17,29,33}$ In our study, EDT was used as a substitute for the OA ligand (see the schematic illustration in Figure 1a). For the EDT ligand-exchange process, methanol (MO) or acetonitrile (AN) solvents were used. The optical properties varied significantly with the capping ligands, as shown in the Fourier-transform infrared 


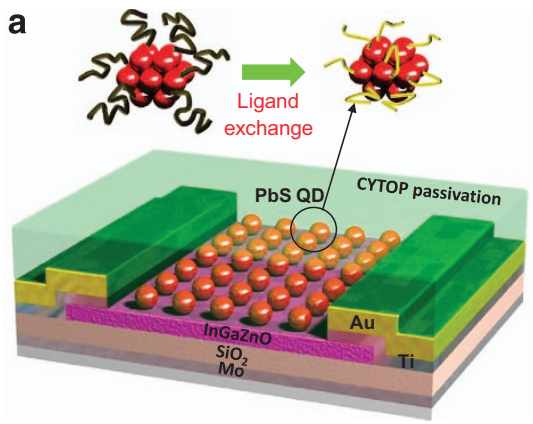

b
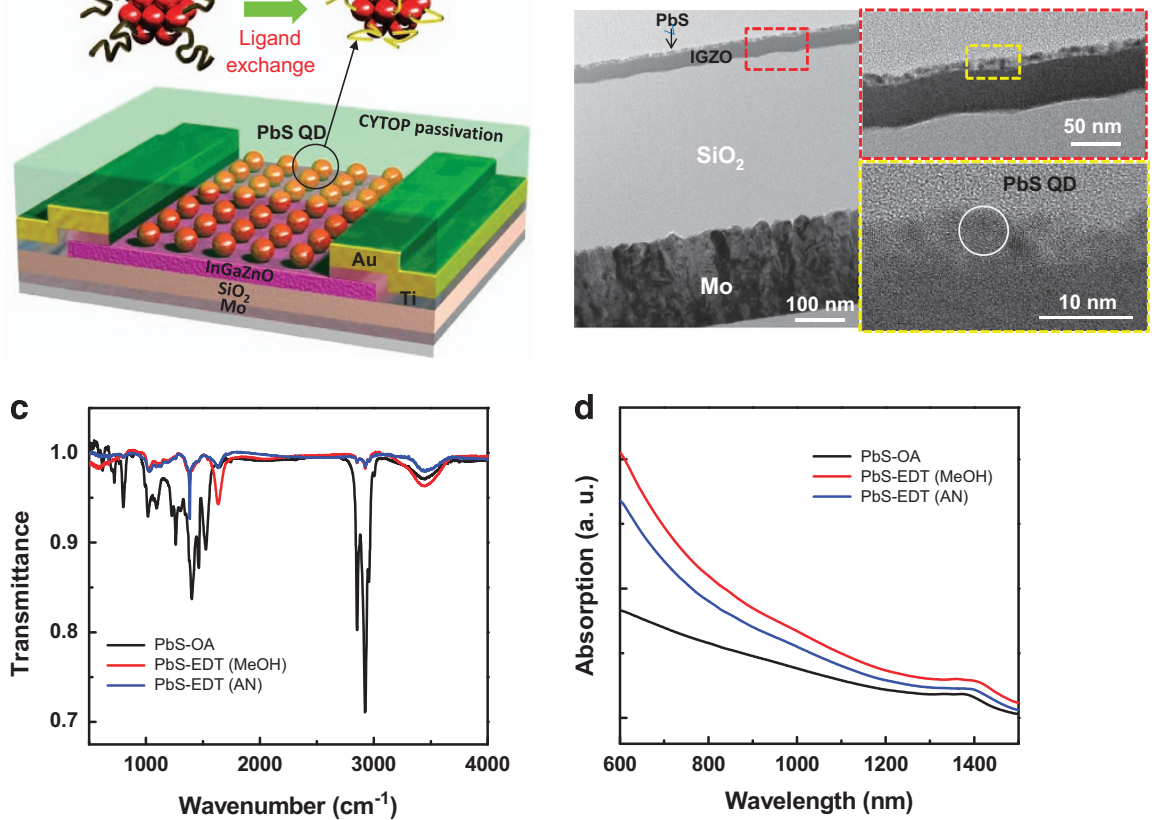

Figure 1 Device structure and the ligand exchange process. (a) A schematic 3D view of the ligand exchange process and the PbS QD/IGZO hybrid phototransistor. (b) Corresponding cross-sectional HRTEM images. (c) FTIR spectra and (d) absorption spectra of the pristine PbS capped with the OA ligand film and the EDT-treated films processed with MO and AN solvents. EDT, ethanedithiol; FTIR, Fourier transform infrared; IGZO, indium gallium zinc oxide; HRTEM, high-resolution transmission electron microscopy; PbS, lead sulfide; QD, quantum dots.

spectra of Figure 1c and the visible-near infrared absorption spectra of Figure 1d. The MO solvent is likely to be more efficient for the EDT ligand-exchange process than the AN solvent, which has a significant impact on the photoelectrical performance of the device. Additional details on the effect of the ligand-exchange process on optical properties are described in the Supplementary Information.

\section{Device performance}

Figures $2 \mathrm{a}-\mathrm{c}$ show the representative transfer and output characteristics of IGZO, PbS-OA/IGZO and PbS-EDT (processed with MO)/ IGZO TFTs $(W / L=1000 \mu \mathrm{m} / 50 \mu \mathrm{m})$ measured in the dark and in air. We fabricated three types of IGZO (IGZO, PbS-OA/IGZO, and PbSEDT/IGZO) TFTs in order to investigate the photodetecting properties simultaneously. All devices were measured with CYTOP passivation for fair comparison. The sole IGZO exhibited negligible hysteresis and achieved an averaged mobility $(\mu)$ value of $16.70 \pm 2.59 \mathrm{~cm}^{2} \mathrm{~V}^{-1} \mathrm{~s}^{-1}$ with $V_{\text {th }}$ of $3.88 \pm 0.83 \mathrm{~V}$ and an on/off current $\left(I_{\text {on }} / I_{\text {off }}\right)$ ratio of $10^{8}$ (over 16 devices on the same substrate). The PbS-OA/IGZO TFTs showed comparable performances s: average values (over 16 devices on the same substrate) of $12.61 \pm 0.28 \mathrm{~cm}^{2} \mathrm{~V}^{-1} \mathrm{~s}^{-1}$ for $\mu,-1.79 \pm 0.46 \mathrm{~V}$ for $V_{\mathrm{th}}$, and $10^{8}$ for $I_{\mathrm{on}} / I_{\text {off }}$ ratio were obtained. In PbS-EDT/IGZO TFTs, similar performance parameter values (averaged over 16 devices on the same substrate) of $13.10 \pm 0.15 \mathrm{~cm}^{2} \mathrm{~V}^{-1} \mathrm{~s}^{-1}$ for $\mu, 1.68 \pm 0.86 \mathrm{~V}$ for $V_{\mathrm{th}}$, and $10^{8}$ for $I_{\text {on }} / I_{\text {off }}$ ratio were also observed; nevertheless, the EDT treatment had induced slight hysteresis. PbS-EDT (Processed with AN)/IGZO TFTs were also fabricated, in which the performance parameter values were not significantly different (see Supplementary Figures S2a and S2b for the representative transfer and output characteristics in the Supplementary Information). All three types of IGZO, PbS-OA/IGZO, and PbS-EDT/IGZO TFTs had good electrical performances and device-to-device uniformity. In order to sequentially monitor the $V_{\text {th }}$ of IGZO TFTs after the PbS-EDT treatment and further CYTOP passivation on the same substrate, a new batch of IGZO TFTs was fabricated. The PbS-EDT treatment induced positive shifts of the turnon and threshold voltages, which were attributed to the formation of the PbS QD and IGZO heterojunction. After the CYTOP passivation, these shifts recovered fractionally. Although the cause of this recovery is not clear, it may be related to the reduction of surface defects, given that hysteresis is also reduced (see Supplementary Figures S2c and S2d). Consequently, the PbS-EDT treatment and CYTOP passivation did not substantially affect the electrical performance of pristine IGZO TFTs despite the threshold voltage shifts.

\section{Phototransistor characteristics}

To investigate the photosensing properties of our devices, the photoinduced transfer characteristics were measured using the photoexcited charge collection spectroscopy system ${ }^{26}$, which consists of a light source of $500 \mathrm{~W} \mathrm{Hg}(\mathrm{Xe})$ arc lamp, a grating monochromator, an optical fiber as an optical probe, and a semiconductor parameter analyzer. The photoinduced transfer curves were obtained under the illumination of monochromatic lights with the spectral range of $700-1500 \mathrm{~nm}$ (at a wavelength interval of $100 \mathrm{~nm}$ ). To measure the incident optical power on the sample at each wavelength, a ultraviolet-enhanced silicon photodetector (Newport, Irvine, CA, USA, 918D-UV-OD3), a Germanium photodetector (Newport, 918D-IR-OD3) and an optical power meter (Newport, 842-PE (R2)) were used. The illumination intensities in this spectral range were measured in the ranges of 0.1 to $0.5 \mathrm{~mW} \mathrm{~cm}^{-2}$ (see Supplementary Figure S3). Figures $3 \mathrm{a}-\mathrm{c}$ display the dark and selected photoinduced transfer curves of IGZO, PbS-OA/ IGZO and PbS-EDT/IGZO TFTs (note: the full-range photoinduced transfer curves are shown in Supplementary Figure S4). The IGZO TFTs showed negligible photosensitivity to wavelengths in the NIR 

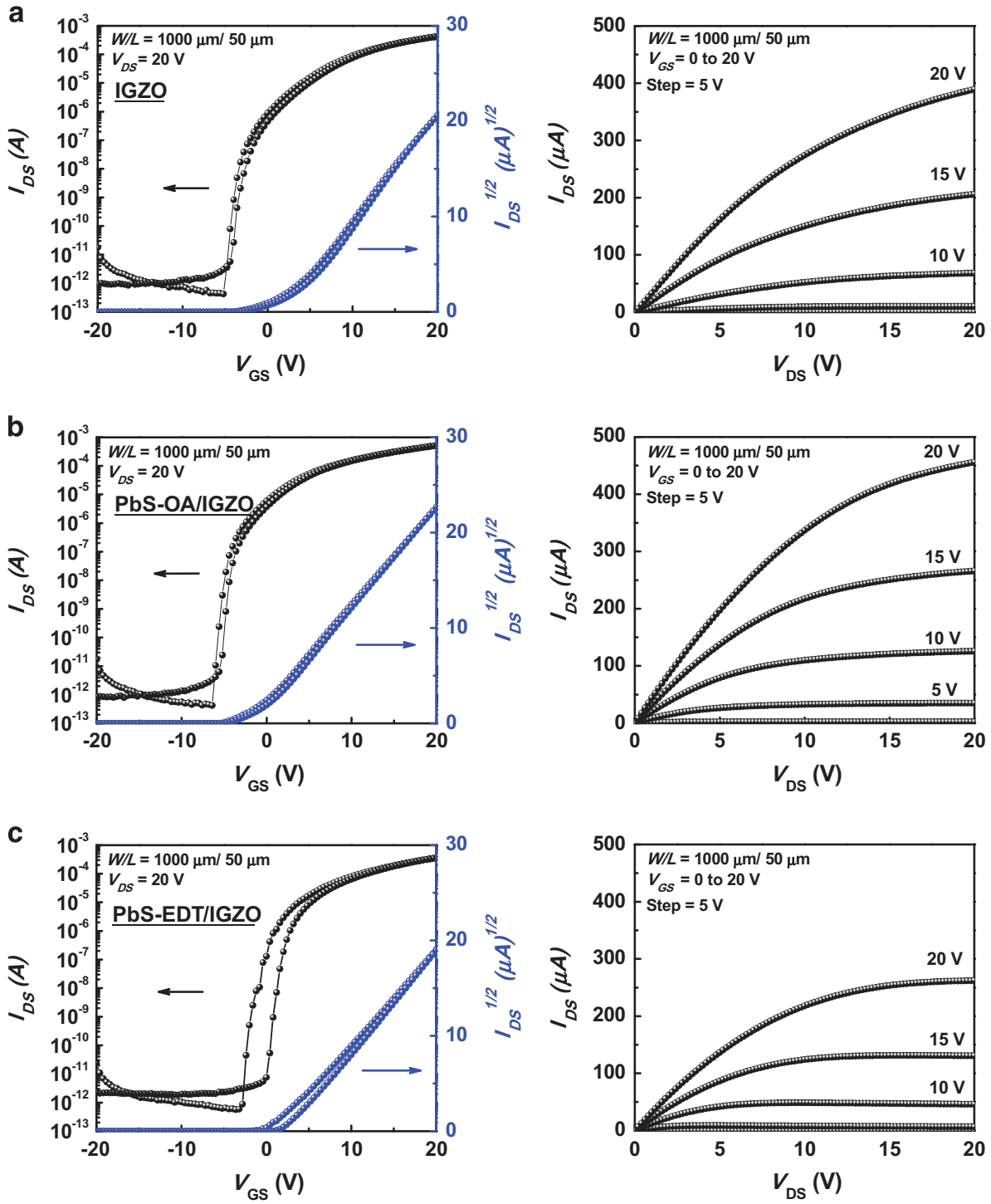

Figure 2 Representative transfer and output characteristics of (a) IGZO, (b) PbS-OA/IGZO, and (c) PbS-EDT (processed with MO)/IGZO TFTs $(W / L=1000 \mu \mathrm{m}$ per $50 \mu \mathrm{m})$ measured in the dark and in air. EDT, ethanedithiol; IGZO, indium gallium zinc oxide; MO, methanol; OA, oleic acid; $\mathrm{PbS}$, lead sulfide; TFTs, thin-film transistors.

spectral region (Figure 3a) due to the wide energy bandgap of $3.4 \mathrm{eV}$ (corresponding to the wavelength of $364 \mathrm{~nm}$ ), and, as in our previous study, its defect energy levels responded to photons over $2.0 \mathrm{eV}$ (corresponding to the wavelength of $620 \mathrm{~nm}$ ). ${ }^{34}$ As seen in Figure $3 \mathrm{~b}$, the PbS-OA/IGZO TFTs did not show significant photoresponsive behavior, indicating that the long alkyl chains block the pathway of the photogenerated charge carrier transfer between $\mathrm{PbS}$ QD and the IGZO layer. In contrast, the PbS-EDT (Processed with MO)/IGZO TFTs exhibited photodetection capabilities for NIR light up to $1400 \mathrm{~nm}$ (see Figure 3c), which matched the absorption spectra of the PbS-EDT film well. As the wavelength of the illuminating light decreased from 1500 to $700 \mathrm{~nm}, V_{\text {th }}$ negatively shifted, with the difference reaching $>10 \mathrm{~V}$ at $700 \mathrm{~nm}$. Upon illumination with light with energy larger than the bandgap energy of PbS QDs, electron-hole pairs (excitons) are generated. These excitons can be easily dissociated at the $\mathrm{PbS} \mathrm{QD} / \mathrm{IGZO}$ interface under a drain voltage of $20 \mathrm{~V}$. The photogenerated electrons can be efficiently injected to the conduction band of the IGZO layer and contribute to the drain current on the
IGZO channel; and according to the literature, the conduction band minimum (or the lowest unoccupied molecular orbital) of $\mathrm{PbS}$ with a diameter of $5 \mathrm{~nm}$ has been calculated to be $4.3 \mathrm{eV}$, ${ }^{30}$ which matches the conduction band edge of IGZO well. ${ }^{35}$ Consequently, the photogenerated electrons induce significant negative $V_{\text {th }}$ shifts (over $10 \mathrm{~V}$ ), as shown in Figure 3c. However, the photogenerated holes are trapped on the PbS QDs (or at the interface of PbS QDs/ IGZO) due to the large energy barrier (note: the valence bands of PbS and IGZO are estimated to be approximately 5.2 and $7.7 \mathrm{eV}$, respectively), which affect the photoswitching characteristics of the phototransistor. A schematic illustration of photoinduced carrier generation and separation phenomenon is shown in Figure $3 \mathrm{~d}$. Whereas IGZO is a well-known $n$-type material, PbS QD is generally accepted to be an ambi-polar material. However, the CYTOP fluoropolymer passivation layer may induce $p$-type dominant characteristics of $\mathrm{PbS}$ QD because abundant carbon-florin (C-F) dipoles could suppress the $n$-type characteristics. Indeed, in our recent work, we found that the CYTOP passivation layer enhances the p-type 

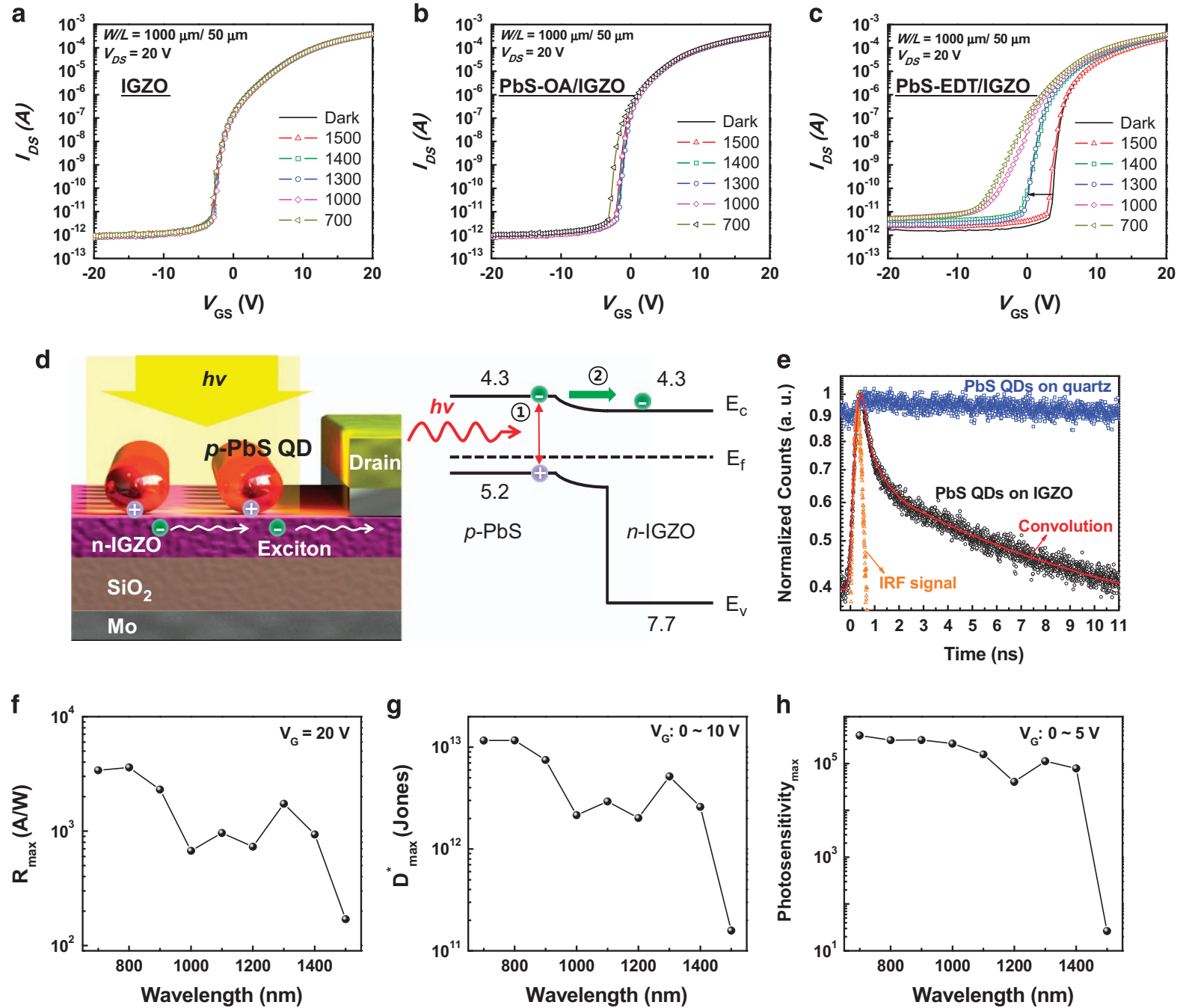

Figure 3 Dark and selected photoinduced transfer curves of (a) IGZO, (b) PbS-OA/IGZO, and (c) PbS-EDT (processed with MO)/IGZO TFTs under monochromatic light with a spectral range of $700-1500 \mathrm{~nm}$. (d) Schematic illustration and energy diagram of the operation mechanism of the phototransistor. The photogenerated electrons from the PbS QDs can be transferred to the conduction band of the IGZO layer, leading to significant negative $V_{\text {th }}$ shifts. However, the photogenerated holes are trapped on the surface of the PbS QDs due to the large energy barrier. (e) Time-resolved photoluminescence trace of the PbS QDs on quartz (blue open square) and IGZO films (black open circle). (f) Maximum values of photoresponsivity, (g) specific detectivity, and (h) photosensitivity of the PbS-EDT phototransistor as a function of illumination wavelength. EDT, ethanedithiol; IGZO, indium gallium zinc oxide; MO, methanol; OA, oleic acid; PbS, lead sulfide; TFTs, thin-film transistors.

characteristics of $\mathrm{WSe}_{2}$ and suppresses the $n$-type characteristics of $\mathrm{MoS}_{2}{ }^{36}$ Similar phenomena have also been observed in organic semiconductors. ${ }^{37-39}$ Although the exact Fermi energy levels and the depletion region lengths of $\mathrm{PbS} \mathrm{QD}$ and IGZO are unknown, the conceptual energy band diagram of the $p$ - $\mathrm{PbS} / n$-IGZO junction in Figure $3 \mathrm{~d}$ illustrated the photogenerated charge transfer processes well. To further clarify this working mechanism, time-resolved photoluminescence (TRPL) experiments using the time-correlated singlephoton counting technique (TCSPC) were carried out. Two samples of $\mathrm{PbS}$ QD single layer on quartz substrate and PbS QD/IGZO bi-layer on glass substrate were prepared. These two samples were excited with $800-\mathrm{nm}$ pulse laser, and TRPL traces were recorded with a probe at $1300 \mathrm{~nm}$. The PL dynamics of the PbS QD single layer and the PbS QD/IGZO bi-layer were significantly different, as shown in Figure 3e. Whereas the TRPL in the PbS QD single-layer film exhibited monoexponential decay dynamics with a few hundred nanosecond time scale, the decay time observed in the PbS QD/IGZO sample was much faster and within laser-pulse repetition (12.5 ns for our Ti: sapphire laser oscillator), indicating that the photogenerated charge carriers from $\mathrm{PbS}$ QDs can be transferred to IGZO films. ${ }^{40-42}$ The PbS QD layer processed with the AN solvent was also applied to make the photosensing layer, but we observed less effective photosensitivity (less negative $V_{\text {th }}$ shifts: see Supplementary Figure S5); this can be directly correlated with the degree of the EDT ligand exchange as previously mentioned in the discussion regarding absorption spectra.

The photoinduced $V_{\text {th }}$ shifts provide additional information regarding the extraction of optical energy bandgap of the PbS QD film. The photoinduced $V_{\text {th }}$ shifts (as a function of the incident photon energy) can be converted to the photoexcited effective charge density $\left(Q_{\text {eff }}\right)$ as shown in the following equation: ${ }^{43}$

$$
\Delta Q_{\text {eff }}(\varepsilon)=C_{\text {ox }} \bullet \Delta V_{\text {th }}(\varepsilon)
$$

where $C_{\mathrm{OX}}$ is the capacitance density of the $\mathrm{SiO}_{2}$ dielectric layer, and $\varepsilon$ is the incident photon energy. The $Q_{\text {eff }}$ plot was obtained from the 
photoinduced transfer curves measured by the photoexcited charge collection spectroscopy system (at a wavelength interval of $20 \mathrm{~nm}$ ) and is shown in Supplementary Figure S6. On the basis of the plot in Supplementary Figure S6, the optical energy bandgap of the PbS-EDT film is found to be $0.86 \mathrm{eV}(\sim 1440 \mathrm{~nm})$ and consistent with the absorption spectra. According to the photoinduced transfer curves of Figure $3 c$, a sudden large $V_{\text {th }}$ shift is observed at a wavelength of $1400 \mathrm{~nm}$ as indicated by an arrow.

Generally, $R$ and $D^{*}$ are figure-of-merits commonly used to evaluate the performance of photodetecting devices. They are given by the equation $R=I_{\mathrm{ph}} / P$ and $D^{*}=(A \Delta f)^{1 / 2} / \mathrm{NEP}$, where $I_{\mathrm{ph}}$ is the photocurrent, $P$ is the incident optical power, $A$ is the active area of the device (channel width $W \times$ channel length $L$ ), $\Delta f$ is the spectral bandwidth (set to 1 in the measurement system for this work), and NEP is the noise equivalent power. The values of $R$ can be directly extracted from the photoinduced transfer curves of the PbS-EDT/ IGZO phototransistor, which are plotted in Supplementary Figure S7. According to Supplementary Figure S7, $R$ increases with the applied gate voltage $\left(V_{\mathrm{G}}\right)$ due to an increase of $I_{\mathrm{ph}}$, and it reaches the maximum value at $V_{\mathrm{G}}=20 \mathrm{~V}$ (device on-state). Similar phenomena have also been observed in organic semiconductors and PbS QD blend device. ${ }^{44}$ Figure $3 \mathrm{e}$ shows the maximum values of $R\left(R_{\max }\right)$ as a function of the illumination wavelength in the spectral region of $1400-700 \mathrm{~nm}$, which range between 670 and $3600 \mathrm{~A} \mathrm{~W}^{-1}$. These values are comparable to or even better than the values of the $\mathrm{PbS}$ QD-based NIR photodetecting devices ${ }^{8}$ or the $2 \mathrm{D}$ nanosheet-based phototransistors ${ }^{45,46}$ previously reported in the literature. To estimate $D^{*}$, a root-mean-square dark noise current of $\left.\left(<I_{n}{ }^{2}\right\rangle\right)^{1 / 2}$ from the noise power spectral densities was measured as a function of $V_{\mathrm{G}}$ using an SR 570 noise current preamplifier (Stanford Research System (SRS)) and an Advantest R9211B digital spectrum analyzer (Advantest Corporation). The gate-dependent noise power spectral densities are shown in Supplementary Figures S8a and S8b. Taking the measured $R$ into account, the gate-dependent $\left.\operatorname{NEP}\left(=\left(<I_{n}{ }^{2}\right\rangle\right)^{1 / 2} / R\right)$ can be calculated (see Supplementary Figure S8c). The active area $(A)$ of the device was $5 \times 10^{-4} \mathrm{~cm}^{2}$. Finally, the gate dependent $D^{*}$ can be estimated, as shown in Supplementary Figure S8d. Unlike $R$, the highest peaks lay between $V_{\mathrm{G}}=0$ and $10 \mathrm{~V}$ (device off-state or subthreshold state). The different tendency is attributed to the fact that $D^{*}$ is inversely proportional to the dark-noise current, which is likely to be more dominant when determining detectivity. The noise power spectral densities increased markedly at the threshold (up to $10^{-18} \mathrm{~A}^{2} \mathrm{~Hz}^{-1}$, as shown in Supplementary Figure S8b), and the optimum detectivity was observed in the sub-threshold region where the $R$ is still high. As shown in Figure 3f, the maximum values of $D^{*}$ were estimated to be $10^{12}-10^{13}$ Jones in the spectral range of 1400 $700 \mathrm{~nm}$. These values are similar to those in the previous reports for state-of-the-art PbS QD-based photodetectors ${ }^{8}$. It is worth noting that the device in this work compares favorably with the commercial Si or InGaAs photodiodes $\left(D^{*} \sim 10^{13}\right.$ Jones $)$, which are generally more sensitive than phototransistors. ${ }^{45,46}$ In addition, the noise power spectral densities in the system background ranged from $10^{-24}$ to $10^{-25} \mathrm{~A}^{2} \mathrm{~Hz}^{-1}$, which were similar to those in a device-off state. According to the literature, ${ }^{47}$ the noise power spectral density of an IGZO transistor (which showed similar electrical performance) can be below $10^{-25} \mathrm{~A}^{2} \mathrm{~Hz}^{-1}$. Therefore, there is room for further improvement of the detectivity by reducing the system background noise.

The photosensitivity, defined as $I_{\mathrm{ph}} / I_{\mathrm{dark}}$, is also a performance parameter of phototransistors. The parameter represents the current on/off ratio induced by the incident light. The photosensitivity behavior is very similar to that of $D^{*}$, which is dependent on $V_{\mathrm{G}}$ with the maximum value between $V_{\mathrm{G}}=0$ and $5 \mathrm{~V}$ (see Supplementary Figure S9). The maximum photosensitivities at each incident wavelength were plotted in Figure 3g, and the values in the order of $10^{5}$ were observed in the spectral range of $1400-700 \mathrm{~nm}$. Considering that the on/off current ratio is on the order of $10^{5}-10^{7}$ in electrical gating in conventional TFTs, the hybrid phototransistor in this work demonstrates the ability to provide proper on/off functions by NIR photogating.

Another important characteristic of phototransistors is the dependence of performance parameters on illumination powers. A fresh IGZO/PbS-EDT device batch was fabricated, and the dependence of its photoresponse on the incident optical power was examined. The monochromatic NIR light with a wavelength of $1000 \mathrm{~nm}$ was selected. To modulate the incident optical power, the optical setup was modified to insert neutral density filters (NIR ND Filter Kit, $25 \mathrm{~mm}$ Dia Filters, \#47-538, Edmund optics, Barrington, NJ, USA). Figure 4a shows the dark and photo-induced transfer curves with different optical powers ranging from $5.97 \mathrm{pW}\left(11.9 \mathrm{nW} \mathrm{cm}^{-2}\right)$ to $0.62 \mathrm{nW}$ $\left(1.28 \mu \mathrm{W} \mathrm{cm}{ }^{-2}\right.$; (note: these incident optical powers were calculated from the measured incident flux in the active area of our samples). Our hybrid phototransistor was found to be very sensitive to low intensity light, even at the lowest illumination power of $5.97 \mathrm{pW}$. The tendency of the photocurrent, responsivity, and detectivity with illumination intensity was also studied. As the light intensity decreased, the photocurrent at $V_{\mathrm{G}}=20 \mathrm{~V}$ was reduced (Figure $4 \mathrm{~b}$ ). In contrast, $R$ and $D^{*}$ at $V_{\mathrm{G}}=20 \mathrm{~V}$ increased with decreasing illumination intensity, reaching $10^{6} \mathrm{~A} \mathrm{~W}^{-1}$ and $3 \times 10^{13}$ Jones as shown in Figures $4 \mathrm{c}$ and $\mathrm{d}$. The values of these performance parameters were similar to those in a previous report for $\mathrm{PbS} \mathrm{QD} / \mathrm{MoS}_{2}$ hybrid phototransistors. ${ }^{32}$ More details on the gate dependent $R, D^{*}$ and NEP as a function of illumination intensity are summarized in Supplementary Figure S10. Here it should be noted that the photocurrent will be saturated and the slope of the tendency will be reduced in higher illumination intensity region, and, as a result, the values of $R$ and $D^{*}$ will be affected. This is attributed to the accumulation of photogenerated charge carriers that will induce reserve electric fields. ${ }^{32}$ The saturation of sensitizing traps in $\mathrm{PbS}$ QDs from photogenerated carriers ${ }^{32}$ can also explain the reduction of photocurrent. ${ }^{12,29}$ This deficiency of linearity can be partially overcome by quickly eliminating the accumulated carriers. Carefully selecting the materials surrounding the PbS QDs to ease the movement of the carriers or introducing new electrical or optical methods to deplete the carriers in the QDs are ways that can eliminate such carriers.

Supplementary Figures S11a and S11b show the temporal photocurrent response of the IGZO/PbS-EDT device. According to relevant literature, ${ }^{29}$ the theoretical photoconductive gain could be calculated from the temporal response, which is in line with the measured gain of $1.24 \times 10^{6}$ (note: $\mathrm{R}$ of $10^{6} \mathrm{~A} \mathrm{~W}^{-1}$ corresponds to photoconductive gain of $1.24 \times 10^{6}$ at a wavelength of $1000 \mathrm{~nm}$ ). From the temporal photocurrent response curve of Supplementary Figure $\mathrm{S} 11 \mathrm{~b}$, we extracted carrier lifetimes of $0.57 \mathrm{~s}$ (fast decay process) and $9.55 \mathrm{~s}$ (slow decay process). For the PbS-EDT/IGZO device, the transient time was found to be $96 \mathrm{~ns}$ (based on an IGZO mobility of $13 \mathrm{~cm}^{2} \mathrm{~V}^{-1} \mathrm{~s}$, an applied $V_{\mathrm{DS}}$ of $20 \mathrm{~V}$, and a channel length of $50 \mu \mathrm{m})$. A theoretical photoconductive gain between $5.9 \times 10^{6}$ and $9.9 \times 10^{7}$ was calculated on the basis of the transient time and carrier lifetimes, which was consistent with measured gain of $1.24 \times 10^{6}$.

\section{Photogating inverter and NIR imaging}

To further evaluate the real potential of the hybrid phototransistor as the basic building blocks for an optoelectronic system, a photogating 

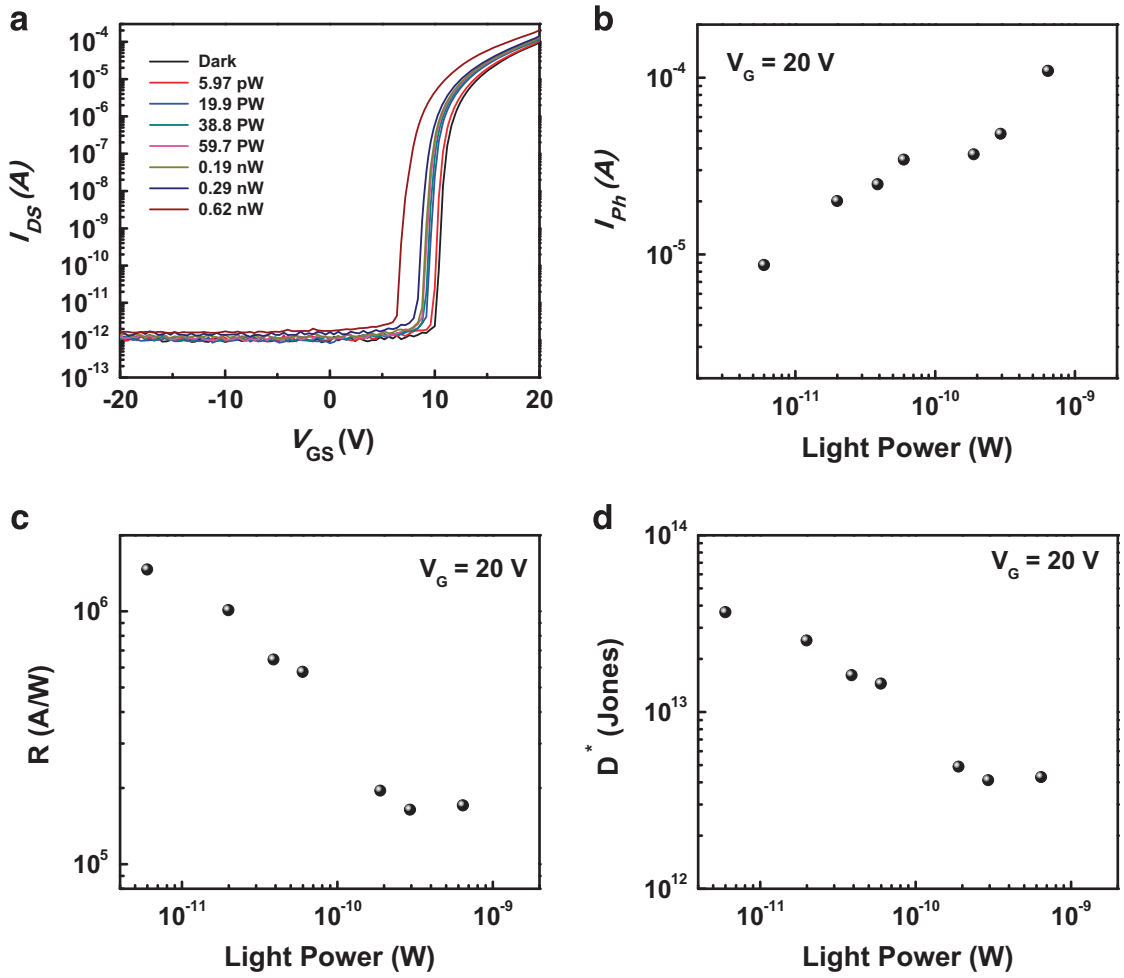

Figure 4 Dependence of photoresponse on illumination intensity. (a) Dark and photoinduced transfer curves under monochromatic light (1000 nm wavelength) with different optical power. (b) Photocurrent, (c) photoresponsivity, and (d) specific detectivity at $V_{\mathrm{G}}=20 \mathrm{~V}$ versus optical illumination power.

resistive-load inverter was implemented by connecting a unit phototransistor to an external load resistor $\left(R_{\mathrm{L}}=100 \mathrm{M} \Omega\right)$. A commercial NIR light emitting diode (LED) with a wavelength of $1300 \mathrm{~nm}$ (SMBB1300-1100, Marubeni America Corporation) was used to measure the photo-induced static and the dynamic characteristics of the photo-inverter; the on/off switching of the light was controlled by a function generator. The photo-induced $V_{\text {th }}$ shift of the hybrid phototransistor produced pronounced output voltage signals in the static and dynamic characteristics of the photo-inverter, as shown in Figures $5 \mathrm{a}-\mathrm{c}$. In the static behavior of the voltage transfer characteristics (VTC), the initial switching threshold voltage $\left(V_{M}\right)$ was $3.7 \mathrm{~V}$, but the photo-induced $V_{M}$ was $0.6 \mathrm{~V}$ under illumination. After the LED was turned off, $V_{M}$ was back to $3.1 \mathrm{~V}$. The photo-inverter also exhibited clear photo-switching voltage dynamics at a fixed input voltage $V_{\text {in }}=2 \mathrm{~V}$ as the LED switched on and off for a period of $10 \mathrm{~s}$ $(0.1 \mathrm{~Hz})$ and $1 \mathrm{~s}(1 \mathrm{~Hz})$; a high output voltage photo-gain of $\sim 4.9 \mathrm{~V}$ was observed between dark and NIR light, as shown in Figures $5 \mathrm{~b}$ and $\mathrm{c}$. In the photo-voltage dynamics, the rising and falling times were estimated to be approximately $0.1-0.2 \mathrm{~s}$ and $0.2-0.3 \mathrm{~s}$, respectively, which are very similar to previously reported values for $\mathrm{PbS} \mathrm{QD} / \mathrm{MoS}_{2}$ hybrid phototransistors. ${ }^{32}$ The relaxation process was not very fast, which could be attributed to the presence of photo-generated holes trapped at the $\mathrm{PbS} \mathrm{QD} / \mathrm{IGZO}$ interface. These trapped holes may lead to a trap-induced current of IGZO electrons (located near hole traps) even in the absence of light, hindering the fast recovery process. However, such relatively slow photodynamic behavior can be enhanced by an additional positive gate voltage pulse, which would lead to a fast reset by the rapid recombination of holes trapped in the PbS QD layer. ${ }^{20,29,46}$

Using the above photo-gating inverter, the prototype NIR imager with a single pixel was demonstrated. Figure $5 \mathrm{~d}$ shows the schematic illustration of the image scanning system and a photograph of the NIR imager. The NIR (1300 nm wavelength) LED was located behind the metallic IR shadow mask carved with the 'KIST' logo. On the other side, the photogating inverter assembled with an $x-z$ planar moving stage (VEXTA C7214-9015-1, Oriental Motor) was placed in proximity to the IR shadow mask. More detailed schematic of the experimental setup for the NIR imaging process is shown in Supplementary Figure S12. Our single pixel imager scans the original image of the 'KIST' logo in the $\mathrm{x}$ and $\mathrm{z}$ directions for a period of $1 \mathrm{~s}$ $(1 \mathrm{~Hz})$. The scan ranges of the $\mathrm{x}$ and $\mathrm{z}$ directions were $20 \mathrm{~mm}$ in $200 \mu \mathrm{m}$ increments and $12 \mathrm{~mm}$ in $500 \mu \mathrm{m}$ increments, respectively. Figure $5 \mathrm{e}$ shows the photograph of the IR shadow mask with the 'KIST' logo (top) and the corresponding output images (bottom) acquired with the NIR imager. Owing to the limitation of the scan range, two scans were executed, one each for 'KI' and 'ST'. To confirm the NIR imaging results, the visible-NIR light below $1200 \mathrm{~nm}$ wavelength was filtered out by placing the $\mathrm{Si}$ wafer between the metallic shadow mask and the NIR imager. Even though the image was duplicated due to multiple reflections within the Si wafer, the ' $K$ ' was clearly observed. More details on the schematic illustration of the NIR imaging process with the Si wafer used as a vis-NIR filter (cut-off below $1200 \mathrm{~nm}$ wavelength) and the measured image are shown in Supplementary Figure S13.

\section{CONCLUSIONS}

We introduced a new approach to fabricate $\mathrm{PbS}$ QD/IGZO hybrid phototransistors for cost-effective NIR detection. The PbS NIR-sensitized layer was easily formed on top of a prefabricated IGZO TFT array by a facile spin-coating process. To enhance the charge transfer characteristics between $\mathrm{PbS}$ QDs and the IGZO channel, the OA ligand with long alkyl chains was replaced with the 

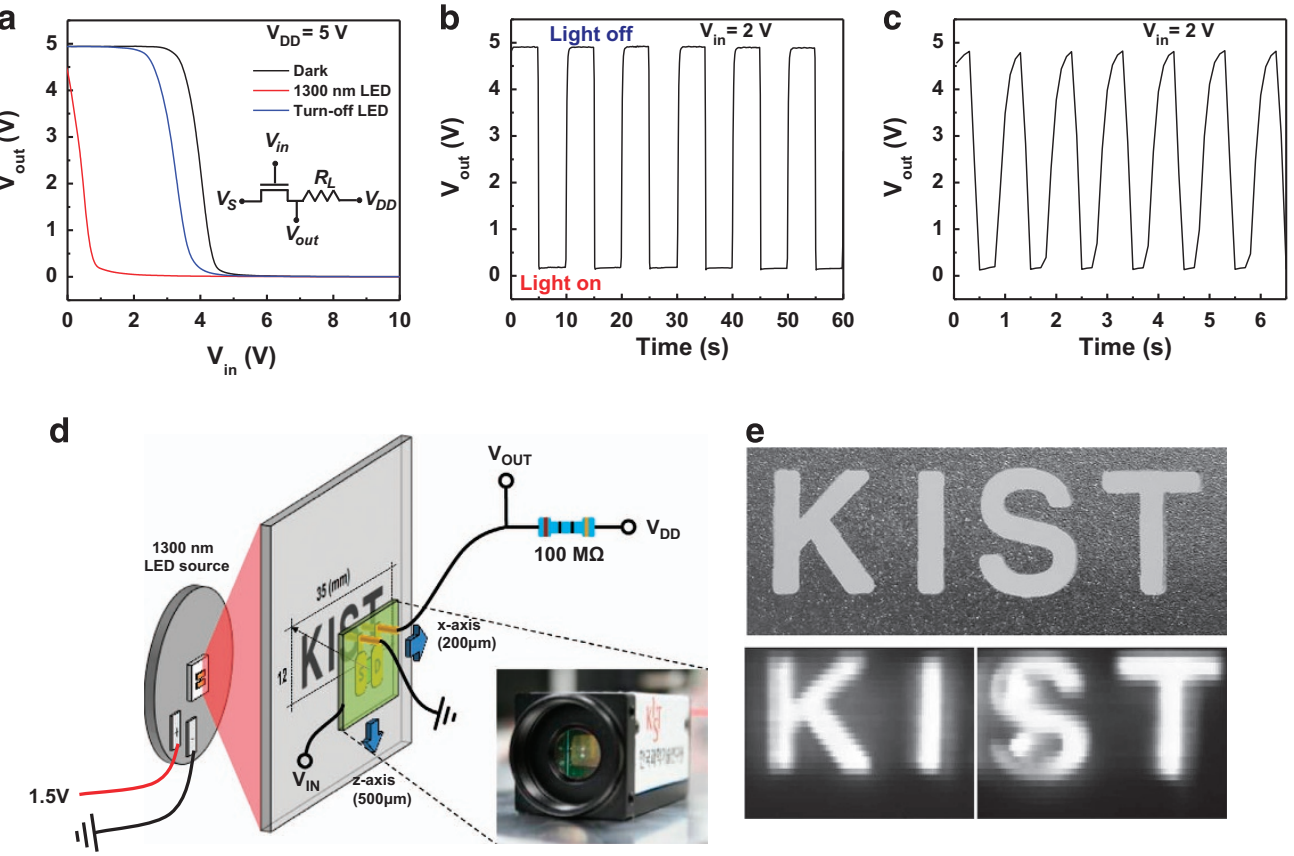

Figure 5 Photogating inverter and NIR imaging. (a) Photoinduced (and dark) voltage transfer characteristics of the resistive-load photoinverter, which is constructed by connecting a unit phototransistor to an external load resistor $\left(R_{\mathrm{L}}=100 \mathrm{M} \Omega\right)$ (see the inset of the circuit diagram), under NIR light from an LED (1300 nm wavelength). (b) Photoswitching voltage dynamics at fixed input voltage $V_{\text {in }}=2 \mathrm{~V}$ under NIR LED switching on/off for a period of $10 \mathrm{~s}$ and (c) $1 \mathrm{~s}$. (d) Schematic illustration of the image scanning system and a photograph of the PbS/IGZO phototransistor-based NIR imager. (e) Original image made from a metal shadow mask and corresponding image acquired with an NIR (1300 nm wavelength) imager. IGZO, indium gallium zinc oxide; LED, light-emitting diode; NIR, near infrared; PbS, lead sulfide.

shorter EDT ligand by a ligand exchange process. The PbS-EDT/IGZO phototransistor then displayed much improved photosensing capabilities for NIR light up to $1400 \mathrm{~nm}$, which cannot be covered by $\mathrm{Si}$ detectors. The phototransistor had an excellent spectral photoresponsivity of over $10^{3} \mathrm{~A} \mathrm{~W}^{-1}$ and a specific detectivity on the order of $10^{12}-10^{13}$ Jones because the photogenerated electrons from the $\mathrm{PbS}$ sensitized layer were effectively transferred to the conduction band of the IGZO layer. Moreover, the device had high sensitivity and was able to detect NIR (1000-nm wavelength) light, even at a very low power of $5.97 \mathrm{pW}$, with a photoresponsivity of over $10^{6} \mathrm{~A} \mathrm{~W}^{-1}$ and a specific detectivity of over $10^{13}$ Jones. The photoinduced threshold voltage shift of the hybrid phototransistor was again demonstrated by the photoinverter, and the output photovoltage signals were clearly extracted both statically and dynamically with a high output voltage photogain of $\sim 4.9 \mathrm{~V}$. The prototype single pixel NIR (1300 nm wavelength) imager using a unit photoinverter was also demonstrated. On the basis of these results, it has been shown that the approach of combining PbS QDs and IGZO transistors is a promising and practical way to create a gate-tunable, highly sensitive large area flat panel NIR detector/imager on glass or plastic substrates that can be easily integrated. As a low cost substitute for expensive InGaAs-based NIR devices, the QD/metal oxide hybrid NIR detector/imager are expected to expand the optoelectronic application market in the NIR wavelength region.

\section{CONFLICT OF INTEREST}

The authors declare no conflict of interest.

\section{ACKNOWLEDGEMENTS}

DKH, JDS and WKC appreciate the financial support from the KIST Institution Program (No. 2V04010 and 2E25800). SI acknowledges the financial support from NRF (NRL program: Grant No. 2014R1A2A1A01004815), the NanoMaterials Technology Development Program (Grant No. 2012M3A7B4034985), and the Brain Korea 21 plus Program.

1 Caruge, J. M., Halpert, J. E., Wood, V., Bulovic, V. \& Bawendi, M. G. Colloidal quantum-dot light-emitting diodes with metal-oxide charge transport layers. Nat. Photon. 2, 247-250 (2008).

2 Cho, K. S., Lee, E. K., Joo, W. J., Jang, E., Kim, T. H., Lee, S. J., Kwon, S. J., Han, J. Y., Kim, B. K., Choi, B. L. \& Kim, J. M. High-performance crosslinked colloidal quantum-dot light-emitting diodes. Nat. Photon. 3, 341-345 (2009).

3 Kim, T. H., Cho, K. S., Lee, E. K., Lee, S. J., Chae, J., Kim, J. W., Kim, D. H., Kwon, J. Y., Amaratunga, G., Lee, S. Y., Choi, B. L., Kuk, Y., Kim, J. M. \& Kim, K. Full-colour quantum dot displays fabricated by transfer printing. Nat. Photon. 5, 176-182 (2011).

4 Son, D. I., Kwon, B. W., Park, D. H., Seo, W. S., Yi, Y., Angadi, B., Lee, C. L. \& Choi, W. K. Emissive ZnO-graphene quantum dots for white-light-emitting diodes. Nat. Nanotechnol. 7, 465-471 (2012).

5 McDonald, S. A., Konstantatos, G., Zhang, S. G., Cyr, P. W., Klem, E. J. D., Levina, L. \& Sargent, E. H. Solution-processed PbS quantum dot infrared photodetectors and photovoltaics. Nat. Mater. 4, 138-142 (2005).

6 Konstantatos, G., Howard, I., Fischer, A., Hoogland, S., Clifford, J., Klem, E., Levina, L. \& Sargent, E. H. Ultrasensitive solution-cast quantum dot photodetectors. Nature $\mathbf{4 4 2}$, 180-183 (2006)

7 Clifford, J. P., Konstantatos, G., Johnston, K. W., Hoogland, S., Levina, L. \& Sargent, E. H. Fast, sensitive and spectrally tuneable colloidal quantum-dot photodetectors. Nat. Nanotechnol. 4, 40-44 (2009).

8 Konstantatos, G. \& Sargent, E. H. Nanostructured materials for photon detection. Nat. Nanotechnol. 5, 391-400 (2010).

9 Prins, F., Buscema, M., Seldenthuis, J. S., Etaki, S., Buchs, G., Barkelid, M., Zwiller, V., Gao, Y. N., Houtepen, A. J., Siebbeles, L. D. A. \& van der Zant, H. S. J. Fast and Efficient Photodetection in Nanoscale Quantum-Dot Junctions. Nano Lett. 12, 5740-5743 (2012).

10 Pal, B. N., Robel, I., Mohite, A., Laocharoensuk, R., Werder, D. J. \& Klimov, V. I. High-sensitivity p-n junction photodiodes based on $\mathrm{PbS}$ nanocrystal quantum dots. Adv. Funct. Mater. 22, 1741-1748 (2012).

11 Boeberl, M., Kovalenko, M. V., Gamerith, S., List, E. J. W. \& Heiss, W. Inkjet-printed nanocrystal photodetectors operating up to $3 \mathrm{mu}$ m wavelengths. Adv. Mater. 19, 3574-3578 (2007). 
12 Konstantatos, G., Clifford, J., Levina, L. \& Sargent, E. H. Sensitive solution-processed visible-wavelength photodetectors. Nat. Photon. 1 531-534 (2007).

13 Semonin, O. E., Luther, J. M., Choi, S., Chen, H. Y., Gao, J. B., Nozik, A. J. \& Beard, M. C. Peak external photocurrent quantum efficiency exceeding $100 \%$ via MEG in a quantum dot solar cell. Science 334, 1530-1533 (2011).

14 Tang, J., Kemp, K. W., Hoogland, S., Jeong, K. S., Liu, H., Levina, L., Furukawa, M., Wang, X. H., Debnath, R., Cha, D. K., Chou, K. W., Fischer, A., Amassian, A., Asbury, J. B. \& Sargent, E. H. Colloidal-quantum-dot photovoltaics using atomic-ligand passivation. Nat. Mater. 10, 765-771 (2011)

15 Willis, S. M., Cheng, C., Assender, H. E. \& Watt, A. A. R. The Transitional heterojunction behavior of $\mathrm{PbS} / \mathrm{ZnO}$ colloidal quantum dot solar cells. Nano Lett. 12 1522-1526 (2012).

16 Etgar, L., Zhang, W., Gabriel, S., Hickey, S. G., Nazeeruddin, M. K., Eychmuller, A., Liu, B. \& Gratzel, M. High efficiency quantum dot heterojunction solar cell using anatase (001) $\mathrm{TiO}_{2}$ nanosheets. Adv. Mater. 24, 2202-2206 (2012).

17 Tang, J., Brzozowski, L., Barkhouse, D. A. R., Wang, X. H., Debnath, R., Wolowiec, R., Palmiano, E., Levina, L., Pattantyus-Abraham, A. G., Jamakosmanovic, D. \& Sargent, E. H. Quantum dot photovoltaics in the extreme quantum confinement regime: the surface-chemical origins of exceptional air- and light-stability. ACS Nano 4 869-878 (2010)

18 Ma, W. L., Swisher, S. L., Ewers, T., Engel, J., Ferry, V. E., Atwater, H. A. \& Alivisatos, A. P. Photovoltaic performance of ultrasmall PbSe quantum dots. ACS Nano $\mathbf{5}$, 8140-8147 (2011).

19 Rauch, T., Boberl, M., Tedde, S. F., Furst, J., Kovalenko, M. V., Hesser, G. N., Lemmer, U., Heiss, W. \& Hayden, O. Near-infrared imaging with quantum-dot-sensitized organic photodiodes. Nat. Photon. 3, 332-336 (2009).

20 Jeon, S., Ahn, S. E., Song, I., Kim, C. J., Chung, U. I., Lee, E., Yoo, I., Nathan, A. Lee, S., Robertson, J. \& Kim, K. Gated three-terminal device architecture to eliminate persistent photoconductivity in oxide semiconductor photosensor arrays. Nat. Mater. 11, 301-305 (2012)

21 Wager, J. F. Transparent electronics. Science 300, 1245-1246 (2003).

22 Nomura, K., Ohta, H., Takagi, A., Kamiya, T., Hirano, M. \& Hosono, H. Room-temperature fabrication of transparent flexible thin-film transistors using amorphous oxide semiconductors. Nature 432, 488-492 (2004).

23 Fortunato, E. M. C., Barquinha, P. M. C., Pimentel, A., Goncalves, A. M. F., Marques, A J. S., Pereira, L. M. N. \& Martins, R. F. P. Fully transparent $\mathrm{ZnO}$ thin-film transistor produced at room temperature. Adv. Mater. 17, 590-594 (2005).

24 Park, S. H. K., Hwang, C. S., Ryu, M., Yang, S., Byun, C. Shin, J., Lee, J. I., Lee, K. Oh, M. S. \& Im, S. Transparent and photo-stable ZnO thin-film transistors to drive an active matrix organic-light-emitting-diode display panel. Adv. Mater. 21, 678-682 (2009).

25 Oh, M. S., Lee, K., Lee, K. H., Cha, S. H., Choi, J. M., Lee, B. H., Sung, M. M. \& Im, S. Transparent photo-stable complementary inverter with an organic/inorganic nanohybrid dielectric layer. Adv. Funct. Mater. 19, 726-732 (2009).

26 Lee, K., Oh, M. S., Mun, S. J., Lee, K. H., Ha, T. W., Kim, J. H., Park, S. H. K. Hwang, C. S., Lee, B. H., Sung, M. M. \& Im, S. Interfacial trap density-of-states in pentacene- and $\mathrm{ZnO}$-based thin-film transistors measured via novel photo-excited charge-collection spectroscopy. Adv. Mater. 22, 3260-3265 (2010).

27 Fortunato, E., Barquinha, P. \& Martins, R. Oxide semiconductor thin-film transistors: a review of recent advances. Adv. Mater. 24, 2945-2986 (2012).

28 Hwang, D. K., Lee, H. S., Lee, Y. J., Kim, H. H., Noh, Y. S., Lee, J., Nam, S. H. Im, S. \& Choi, W. K. Quantum-dot sensitized metal oxide semiconductor hybrid phototransistor for near infrared detection. MRS Fall Meeting. 06.30. http:// www.mrs.org/fall-2013-program-o/ (2013).

29 Konstantatos, G., Badioli, M., Gaudreau, L., Osmond, J., Bernechea, M. de Arquer, F. P. G., Gatti, F. \& Koppens, F. H. L. Hybrid graphene-quantum dot phototransistors with ultrahigh gain. Nat. Nanotechnol. 7, 363-368 (2012).

30 Zhang, D. Y., Gan, L., Cao, Y., Wang, Q., Qi, L. M. \& Guo, X. F. Understanding charge transfer at PbS-decorated graphene surfaces toward a tunable photosensor. Adv. Mater 24, 2715-2720 (2012).

31 Sun, Z. H., Liu, Z. K., Li, J. H., Tai, G. A., Lau, S. P. \& Yan, F. Infrared photodetectors based on CVD-grown graphene and pbs quantum dots with ultrahigh responsivity. Adv. Mater. 24, 5878-5883 (2012).

32 Kufer, D., Nikitskiy, I., Lasanta, T., Navickaite, G., Koppens, F. H. L. \& Konstantatos, G. Hybrid 2D-OD $\mathrm{MoS}_{2}-\mathrm{PbS}$ quantum dot photodetectors. Adv. Mater. 27 176-180 (2015).
33 Jeong, K. S., Tang, J., Liu, H., Kim, J., Schaefer, A. W., Kemp, K., Levina, L., Wang, X. H., Hoogland, S., Debnath, R., Brzozowski, L., Sargent, E. H. \& Asbury, J. B. Enhanced mobility-lifetime products in PbS colloidal quantum dot photovoltaics. ACS Nano 6, 89-99 (2012).

34 Chang, Y. G., Kim, D. H., Ko, G., Lee, K., Kim, J. H. \& Im, S. Trap density of states measured by photon probe on amorphous-InGaZnO thin-film transistors. IEEE Electron Device Lett. 32, 336-338 (2011).

35 Lee, K., Nomura, K., Yanagi, H., Kamiya, T., Ikenaga, E., Sugiyama, T., Kobayashi, K. \& Hosono, $\mathrm{H}$. Band alignment of $\mathrm{InGaZnO}_{4} / \mathrm{Si}$ interface by hard $\mathrm{x}$-ray photoelectron spectroscopy. J. Appl. Phys. 112, 033713 (2012).

36 Jeon, P. J., Min, S.-W., Kim, J. S., Raza, S. R. A., Choi, K., Lee, H. S., Lee, Y. T., Hwang, D. K., Choi, H. J. \& Im, S. Enhanced device performances of $\mathrm{WSe}_{2}-\mathrm{MoS}_{2}$ van der Waals junction p-n diode by fluoropolymer encapsulation. J. Mater. Chem. C 3, 2751 (2015).

37 Kobayashi, S., Nishikawa, T., Takenobu, T., Mori, S., Shimoda, T., Mitani, T., Shimotani, H., Yoshimoto, N., Ogawa, S. \& Iwasa, Y. Control of carrier density by self-assembled monolayers in organic field-effect transistors. Nat. Mater. $\mathbf{3}$ 317-322 (2004)

38 Zschieschang, U., Ante, F., Schloerholz, M., Schmidt, M., Kern, K. \& Klauk, H. Mixed self-assembled monolayer gate dielectrics for continuous threshold voltage control in organic transistors and circuits. Adv. Mater. 22 4489-4493 (2010).

39 Baeg, K.-J., Khim, D., Jung, S.-W., Kang, M., You, I.-K., Kim, D.-Y., Facchetti, A. \& Noh, Y.-Y. Remarkable enhancement of hole transport in top-gated n-type polymer field-effect transistors by a high-k dielectric for ambipolar electronic circuits. Adv. Mater. 24, 5433-5439 (2012).

40 Leventis, H. C., O'Mahony, F., Akhtar, J., Afzaal, M. O'Brien, P.\& Haque, S. A. Transient optical studies of interfacial charge transfer at nanostructured metal oxide/PbS quantum dot/organic hole conductor heterojunctions. J. Am. Chem. Soc. 132, 2743-2750 (2010).

41 Szendrei, K., Cordella, F., Kovalenko, M. V., Boeberl, M., Hesser, G., Yarema, M., Jarzab, D., Mikhnenko, O. V., Gocalinska, A., Saba, M., Quochi, F., Mura, A., Bongiovanni, G., Blom, P. W. M., Heiss, W. \& Loi, M. A. Solution-processable nearIR photodetectors based on electron transfer from $\mathrm{PbS}$ nanocrystals to fullerene derivatives. Adv. Mater. 21, 683-687 (2009).

42 Yakunin, S., Dirin, D. N., Protesescu, L., Sytnyk, M., Tollabimazraehno, S., Humer, M., Hackl, F., Fromherz, T., Bodnarchuk, M. I., Kovalenko, M. V. \& Heiss, W. High infrared photoconductivity in films of arsenic-sulfide-encapsulated lead-sulfide nanocrystals. ACS Nano 8, 12883-12894 (2014).

43 Lee, H. S., Min, S. W., Chang, Y. G., Park, M. K., Nam, T., Kim, H., Kim, J. H., Ryu, S. \& Im, S. $\mathrm{MoS}_{2}$ nanosheet phototransistors with thickness-modulated optical energy gap. Nano Lett. 12, 3695 (2012)

44 Sun, Z., Li, J. \& Yan, F. Highly sensitive organic near-infrared phototransistors based on poly (3-hexylthiophene) and PbS quantum dots. J. Mater. Chem. 22, 21673-21678 (2012)

45 Choi, W., Cho, M. Y., Konar, A., Lee, J. H., Cha, G. B., Hong, S. C., Kim, S., Kim, J., Jena, D. \& Joo, J. High-detectivity multilayer $\mathrm{MoS}_{2}$ phototransistors with spectral response from ultraviolet to infrared. Adv. Mater. 24, 5832 (2012).

46 Lopez-Sanchez, O., Lembke, D., Kayci, M., Radenovic, A. \& Kis, A. Ultrasensitive photodetectors based on monolayer $\mathrm{MoS}_{2}$. Nat. Nanotechnol. 8, 497 (2013).

47 Park, J. C., Kim, S. W., Kim, C. J., Kim, S., Kim, D. H., Cho, I. T. \& Kwon, H. I. Low-frequency noise in amorphous indium-gallium-zinc oxide thin-film transistors from subthreshold to saturation. Appl. Phys. Lett. 97, 122104 (2010).

This work is licensed under a Creative Commons Attribution 4.0 International License. The images or other third party material in this article are included in the article's Creative Commons license, unless indicated otherwise in the credit line; if the material is not included under the Creative Commons license, users will need to obtain permission from the license holder to reproduce the material. To view a copy of this license, visit http://creativecommons.org/licenses/by/4.0/ 